Revista Calidad en la Educación Superior

Programa de Autoevaluación Académica

Universidad Estatal a Distancia, Costa Rica

ISSN 1659-4703

revistacalidad@uned.ac.cr

\title{
INTERACCIÓN DOCENTE EN LA NUBE
}

PROFESSORS INTERACTING IN THE CLOUD

Gabriel Rubén Feldman, gfeldman@herrera.unt.edu.ar ${ }^{1}$
Eduardo Juarez,_ejuarez@face.unt.edu.ar ${ }^{2}$
Universidad Nacional de Tucumán, Argentina

Volumen 7 , Número 1

Mayo 2016

pp. $233-251$

Recibido: 06 de diciembre, 2015

Aprobado: 28 de abril, 2016

\footnotetext{
${ }^{1}$ Contador Público Nacional, Magister en Disciplinas Bancarias. Profesor Asociado de Finanzas de Empresas I, Universidad Nacional de Tucumán (U.N.T.). Investigador del Consejo de Investigaciones de la U.N.T (CIUNT). Argentina.

2 Contador Público Nacional, Profesor Titular de Computación, U.N.T. Director de programa de investigación de CIUNT. Director de la Especialización en Administración Pública (U.N.T.). Argentina
} 


\title{
Resumen
}

La utilización de Tecnología de la Información y la Comunicación (TIC) en el proceso de enseñanza-aprendizaje de las disciplinas de administración ha crecido de manera acelerada. Asimismo, los trabajos de investigación que evalúan la utilidad de su uso, incluyendo la referencia a programas, simuladores, metodologías, talleres y plataformas, que puedan ser adaptados y adoptados según la necesidad. Todos estos avances descriptos se enfocan en la interacción profesor-alumno. El presente trabajo está abocado a un ámbito que no ha tenido igual evolución hasta el momento tanto en su faz empírica ni académica: la interactividad entre quienes tienen a su cargo la puesta en práctica de la tarea docente, es decir interacción profesor-profesor.

Palabras Clave: Conectividad, computación en nube, trabajo en equipo.

\begin{abstract}
The use of Information and Communication Technology (ICT) in the teaching-learning process in the disciplines of administration has grown rapidly. Also the research that evaluates the usefulness of its use, including the reference to programs, simulators, methodologies, workshops and platforms, which can be adapted and adopted as required. All this refers to the professor-student interaction. This paper is aimed at a field that has not had the same evolution so far, both in its academic and empirical face: the interactivity among those responsible for the implementation of the teaching task, that is to say interaction professor-professor.
\end{abstract}

Keywords: Connectivity, cloud computing, teamwork.

\section{Introducción}

La llegada de internet como una importante herramienta de comunicación, motivó la implementación de aplicaciones en línea que catapultaron a la tecnología educativa como una mejor forma en el proceso de aprendizaje.

Pero la vinculación profesor-alumno no es objeto del presente artículo. Lo que sí nos ocupa es contribuir a que el trabajo interactivo grupal de los docentes o instructores opere y coopere hacia una mejora en el resultado final, cualquiera que sea su objetivo. En este contexto, internet ofrece oportunidades sin precedentes para que los profesores compartan experiencias y colaboren con otros centros educativos y áreas e incluso más allá de sus propias fronteras. 
De hecho, las redes de trabajo online ofrecen plataformas donde poder compartir recursos, lo que conlleva también a una reducción en la cantidad de trabajo de los docentes. También, proporcionan un espacio para el desarrollo profesional de los profesores, así como una plataforma para integrar el panorama diverso y fragmentado del aprendizaje a lo largo de la vida.

Es preciso desarrollar los siguientes conceptos para analizar su implementación:

1. Estructura organizativa: Inclusión del proyecto en los programas académicos, sus vínculos con otras áreas, alcance y nivel de intervención en la toma de decisiones, sus principales funciones, la estructura interna en la que operará para alcanzar los objetivos, y las características y personas responsables. Destacamos este último ítem: la figura del líder resulta clave en todo proceso de trabajo grupal.

2. Infraestructura: Equipos disponibles (computadoras, servidores, redes, conexiones satelitales, etc.) así como instalaciones físicas (salas de estudio, aulas virtuales, auditorios, etc.).

3. Dimensión pedagógica del proyecto: El trabajo de plantear, desarrollar y evaluar la curricula de las cuestiones bajo estudio, las técnicas con que se implementarán, y los procedimientos de evaluación.

4. Presupuesto: Provisión de equipamiento y mantenimiento, el personal involucrado, tiempo y recursos destinados para la capacitación de profesores.

El objetivo del presente trabajo es contribuir a desarrollar la habilidad de trabajo en equipo en la docencia universitaria, con la utilización de TIC, como un medio para optimizar la labor académica. Se tratará la temática integrando aspectos de relaciones humanas y tecnológicas, y su aplicación a casos concretos. Se describirán los fundamentos teóricos y metodológicos que sustentan la habilidad de trabajo en equipo en la docencia universitaria y el estado actual de éste en la docencia universitaria. 


\section{Computación en la Nube}

De acuerdo con Joyanes Aguilar (2012) en computación se entiende por trabajar en la nube (Cloud computing) al modelo de gestión de la información y documentación, en servicios y plataformas a través de internet. Es decir, un modelo que permite el acceso bajo demanda por medio de la red a un conjunto compartido de recursos de computación configurables (redes, servidores, almacenamiento, aplicaciones y servicios) que se pueden aprovisionar rápidamente con el mínimo esfuerzo de gestión o interacción del proveedor del servicio.

El término Web 2.0 se viene empleando para designar a una nueva generación de servicios web que se caracterizan fundamentalmente por fomentar la participación de los usuarios y la comunicación entre ellos (Arroyo Vázquez, 2007). Subraya un cambio de paradigma sobre la concepción de internet y sus funcionalidades que ahora abandonan su marcada unidireccionalidad y se orientan más a facilitar la máxima interacción entre los usuarios y el desarrollo de redes sociales o tecnologías sociales (Pere Marques, 2013). Tecnológicamente, las aplicaciones Web 2.0 son servicios de internet, por lo que no es necesario tener instalado un software cliente en el ordenador, ya que este proporciona entornos para el desarrollo de redes de centros y profesores donde puedan reflexionar acerca de temas educativos, ayudarse, elaborar y compartir recursos.

Es oportuno describir cuestiones relativas al modelo de computación en la nube considerando sus inconvenientes y problemáticas; es decir, seguridad, privacidad e integridad.

Seguridad: El usuario no sabe exactamente dónde está almacenada la información, mientras que en la computación tradicional sí. Ahora bien, trasladar toda la información a la nube significa confiar la seguridad a terceros, lo que puede ser motivo de preocupación. Una de las críticas más negativas a La Nube es la relativa a la seguridad y el control de los datos (Joyanes Aguilar, 2012). Así también, es necesario considerar aspectos legales: la nube puede ser más segura que un centro de datos tradicional, si bien el método de reforzar la seguridad de la 
información es radicalmente distinto por lo que se deberá adoptar un enfoque basado en riesgos para moverse o migrar a la nube y seleccionar las opciones de seguridad.

Privacidad: Referida a la prevención de divulgación o revelación, intencionada o no, de información no autorizada. En la nube, la confidencialidad radica en garantizar que la información esté accesible únicamente para el personal autorizado, asegurando el ingreso únicamente a personas que cuenten con la debida autorización (Rodas Orellana y Toscano Cruz, 2015). Así pues, una política de confidencialidad define los requisitos para asegurarla, previniendo la divulgación no autorizada de la información.

Integridad: Hace referencia a la corrección y completitud de información en una base de datos (Larios Olivos, 2014). Esta integridad de los datos se debe garantizar en el tránsito y almacenamiento y así también especificarse los medios de recuperación, a partir de errores detectados.

Las ventajas de la computación en la nube pública no están reservadas para las grandes universidades, pueden ser más pronunciadas en las pequeñas entidades que aún no han logrado niveles elevados de informatización o no cuentan y tienen dificultades para incorporar personal con conocimientos adecuados en Tecnologías de la Información, o que se preocupan por su capacidad para proteger los datos. Al contratar a un proveedor de servicios en la nube, que puede ser tal vez otra universidad más grande, la institución pequeña puede adoptar aplicaciones y servicios de punta, y omitir toda una generación de computación académica, con lo cual evitará muchos problemas que describiremos más adelante.

Las instituciones universitarias son organizaciones complejas, en las que el proceso decisorio de contrataciones se ve influenciado por factores políticos, sociales y económicos. La adopción de la computación en la nube plantea muchos de los mismos riesgos y desafíos que la selección de una estrategia de subcontratación más tradicional. Sin embargo, la mayor 
posibilidad de que el proveedor de servicios o sus recursos residan fuera de la competencia legal o territorial, puede agudizar estas inquietudes (Cisco, 2010).

\section{Trabajo en equipo}

Colaborar es trabajar con otros para conseguir objetivos compartidos y explícitos (Medina, 2014). Por lo cual, trabajar en equipo es colaborar organizadamente para obtener un objetivo en común; es decir, aprovechar las experiencias de las otras personas sobre los temas que los demás no conocen, o al menos no conocen en profundidad.

El trabajo en equipo de tipo cooperativo no se limita al hecho de que varias personas trabajen juntas, sino que además, estas deben tratar de conseguir objetivos comunes, de manera que cada miembro del equipo sólo pueda conseguir sus propios objetivos si los demás componentes del grupo logran los suyos, lo que se denomina interdependencia de metas (Laudon y Laudon, 2012). El requisito básico para que esta actividad pueda desarrollarse es la existencia de finalidades compartidas por todos los miembros del grupo.

Los profesores que trabajan en equipo con sus compañeros suelen practicar también esta actividad en el aula con sus alumnos, ya que el trabajo cooperativo no es un proceso técnico, sino una actitud que impregna todos los aspectos y conforma la cultura de la colaboración que se extiende a todos los momentos y situaciones (Montoya, Gil, et al, 2008).

Según Laudon y Laudon (2012), las razones para el trabajo en equipo son:

(a) Nuevo entorno de trabajo: En la actualidad los trabajos en todos los ámbitos requieren una integración entre las partes, así como en el entorno universitario la interdisciplinaridad emerge como modus operandi en la tarea de investigación. El trabajo colaborativo docente apoyará al proceso educativo en el sentido de ir en la misma dirección de lo que ocurre en los ámbitos empresariales/organizacionales. 
(b) Ámbito cambiante: En la actividad empresaria la interacción regional, nacional e internacional marca la tendencia actual y es posible pensar en un mundo académico que en forma coordinada aborde proyectos tanto en disciplinas afines dentro de cada centro universitario local, como organizándose a través de las asociaciones docentes de cada disciplina. La colaboración no se realiza de una manera espontánea, en especial si no hay una cultura de apoyo; por ende, es necesario crear una cultura colaborativa.

(c) Innovación en equipo: Lograr resultados innovadores difícilmente sea el resultado del trabajo individual de una persona, tanto en la empresa como en la academia. El cambio es un proceso grupal y social y la mayoría de estos cambios se derivan de la colaboración entre individuos. Se cree que las prácticas y tecnologías de colaboración sólidas aumentan el ritmo y la calidad de la innovación.

(d) Cultura cambiante: Las nociones populares de la multitud-crowdsourcing, y la sabiduría de las masas- también proveen apoyo cultural para la colaboración y el trabajo en equipo. El concepto crowdsourcing consiste en externalizar tareas; es decir, proponer una tarea en una convocatoria abierta a un grupo masivo de profesionales ajenos a nuestra organización. A partir de la idea de que la comunidad puede construir y crear cosas nuevas, con más facilidad y rapidez que un individuo, se creó el concepto de que la comunidad es la que colabora para lograr algo.

(e) Sinergia: Es de esperar que el resultado del trabajo en equipo sea mayor que aquel que podría alcanzarse sumando los logros individuales.

El trabajo en equipo que proponemos se basa en herramientas y tecnología. Una cultura colaborativa, orientada a equipos no producirá beneficios si no hay sistemas de información funcionando que permitan la colaboración. 
Pueden mencionarse las siguientes categorías de software de colaboración: Mensajería instantánea, escritura colaborativa, revisión/edición colaborativa, compartir archivos, compartir pantallas, conferencias de audio/video, pizarra blanca Esta tecnología de colaboración que acabamos de describir son formas de sobrepasar las limitaciones de tiempo y espacio, y muchas de ellas se aplican en más de un escenario de tiempo/espacio:

Figura 1. Matriz de Tiempo/Espacio de Herramientas de Colaboración

\begin{tabular}{l|l|l}
\hline & Mismo Tiempo - Sincrónicas & $\begin{array}{l}\text { Diferente Tiempo - } \\
\text { Asincrónicas }\end{array}$ \\
\hline $\begin{array}{l}\text { Mismo Lugar - } \\
\text { Colocadas }\end{array}$ & $\begin{array}{l}\text { Interacciones cara a cara } \\
\text { Salas de decisiones, groupware } \\
\text { de una sola pantalla, tabla } \\
\text { compartida, pantallas de pared, } \\
\text { roomware. }\end{array}$ & $\begin{array}{l}\text { Tarea Continua } \\
\text { Salas de equipos, pantalla } \\
\text { pública grande, cooperación y } \\
\text { colaboración en equipos de } \\
\text { trabajo, gestión de proyectos. }\end{array}$ \\
\hline $\begin{array}{l}\text { Distinto Lugar - } \\
\text { Remotas }\end{array}$ & $\begin{array}{l}\text { Interacciones remotas } \\
\text { Videoconferencias, mensajería } \\
\text { instantánea, diagramas/ MUDs/ } \\
\text { mundos virtuales, pantallas } \\
\text { compartidas, editores } \\
\text { multiusuario. }\end{array}$ & $\begin{array}{l}\text { Comunicación + coordinación } \\
\text { Correo electrónico, tableros de } \\
\text { anuncios, blogs, conferencias } \\
\text { asincrónicas, calendarios en } \\
\text { grupo, flujo de trabajo, control de } \\
\text { versiones, wikis. }\end{array}$ \\
\hline
\end{tabular}

Fuente: Laudon, Kenneth C y Laudon Jane P, 2012.

Dificultades del Trabajo en Equipo

La reunión de diversas personas unidas por un propósito común puede no siempre producir los resultados propios de un trabajo en equipo. Es por ello que nuestro propósito es aportar hacia un trabajo en equipo eficaz. Los profesores encuentran dificultades para trabajar en grupo incluso cuando quieren hacerlo; la escasa tradición y preparación que los enseñantes poseen al respecto es el primer escollo. No se trabaja en conjunto cuando los miembros hacen uso de sus aptitudes y posiciones de manera aislada, ni cuando la perspectiva de cada miembro no aporta un valor añadido específico al conjunto. Trabajar en equipo supone 
entender que toda unidad de trabajo pasa por diferentes fases, por una necesaria dinámica. Hay que encontrar el ambiente y ritmo apropiados para cada fase.

Una de las recomendaciones básicas es que el trabajo de innovación educativa se realice en el seno de un equipo docente coordinado y no de manera individual en cada una de las asignaturas. Sin embargo, la realidad de la universidad es que siendo el nivel educativo con más autonomía por parte del profesorado, es donde la coordinación resulta más difícil. Las culturas competitivas de la sociedad y de los ámbitos profesionales, establecen una pugna más o menos abierta entre los objetivos de las personas o los grupos que la componen.

Otro inconveniente es la balcanización. En este caso se trata de un tipo de cooperación que divide y separa a los profesores en subgrupos aislados, y a veces enfrentados, dentro del mismo departamento o centro. La balcanización aparece cuando los profesores se relacionan de forma más estrecha que en la cultura individualista, pero en grupos, prestando su lealtad y vinculando su identidad a colectivos concretos de colegas (Montoya, Gil, et al, 2008).

\section{Redes Docentes}

Una red docente es una comunidad profesional creada para la autoformación o el aprendizaje compartido que se caracteriza por ser iniciativa voluntaria de profesores, con un fuerte compromiso con la innovación, el cambio y la mejora educativa, con metas y propósitos compartidos (Area Moreira, 2009).

El sector docente es justamente el que debería aprovechar sus esfuerzos sinérgicos para potenciar el proceso en toda la línea: si los profesores se embarcan en proyectos colaborativos con otros colegas, entonces también tienen que propiciar el uso de esta estrategia de aprendizaje con sus alumnos. Sería pertinente que planificaran e implementaran actividades de aprendizaje para que los alumnos trabajen colaborativamente no sólo con sus compañeros de clase, sino también con otros alumnos que geográficamente están distantes. 
Lobato y Mandinabetia (2011) señalan que en cuanto al aspecto motivacional, las redes docentes para trabajar de manera coordinada y en equipo coadyuvan hacia el entusiasmo en la tarea docente. Existen docentes que se han sentido motivados por verse respaldados por profesionales expertos en metodologías activas de enseñanza durante este proceso de cambio metodológico.

\section{Metodología - Casos de Aplicación}

En general, todas las disciplinas de las ciencias económicas cuentan con agrupaciones o asociaciones docentes que reúnen a los profesores e investigadores de la materia y cumple la función institucional correspondiente.

El esquema planteado de redes docentes bien podría adaptarse a cualquiera de las disciplinas de las ciencias económicas, en particular de la disciplina administración. Se verifica de este modo el primer gran fundamento de este tipo de redes: estaría formada por profesores con características comunes. Se analizará el caso de aplicación al entorno de docentes de administración financiera de Argentina, materia que ocupa la curricula de las carreras de Contador Público Nacional, y Licenciatura en Administración de Empresas. El espectro de temas que puede abordarse una vez que la red se encuentre operativa es amplio: pedagógicos, técnicos, académicos, institucionales y también tecnológicos. Las herramientas informáticas avanzan en funcionalidad y siembran el camino hacia la posibilidad de la interconexión a la que hacemos referencia en este trabajo. Se observa en la disciplina un fuerte compromiso hacia la innovación, el cambio y la mejora, lo que se materializa con la participación activa de los profesores e investigadores en las actividades, con metas y propósitos compartidos, y un marcado sesgo hacia la excelencia cognitiva y compromiso social.

Los siguientes ítems podrían ser objeto de aplicación de la propuesta; a saber, Interacción en análisis de contenidos; interacción en análisis de metodologías; interacción en análisis de 
bibliografía; interacción en análisis de recursos y herramientas, entre otros. Es decir que, una vez que la red docente comience a funcionar activamente, consideramos que habrá una interacción que coadyuvará hacia la mejora de la calidad educativa.

Es así que la técnica metodológica consistirá en análisis cualitativo de casos de aplicación de trabajo en equipo docente, basado en computación en la nube. Buscamos proponer y analizar el impacto en situaciones de aplicación de la dinámica propuesta, en consistencia con nuestro objetivo general de propiciar la interacción en docencia superior.

Queda planteado para estudios posteriores la posibilidad de establecer un sistema de variables e indicadores que favorezcan el desarrollo y evaluación del trabajo en equipo acorde con las particularidades desde la docencia universitaria. Es decir, cómo establecer un sistema e indicadores para el desarrollo del trabajo en equipo en la docencia universitaria, y qué vías emplear para constatar la validez de los indicadores para el trabajo en equipo en la docencia universitaria.

\section{V.1. Caso 1 - Interacción en Análisis de Contenidos}

Una cuestión por remarcar en el caso específico que se ejemplifica de administración financiera, es considerar la relativa homogeneidad de contenidos entre las curriculas de las materias ofrecidas en las distintas universidades. Por supuesto, hay muchas cuestiones que puntualizar, ya que podrían influir en el contenido de los cursos de: grado o postgrado, introductorio o avanzado, para Contador Público o Licenciatura en Administración, con perfil analítico o cuantitativo. $Y$ también, otras cuestiones que a priori sabemos que marcarían diferenciaciones por motivos diversos: cantidad de alumnos y docentes, ubicación de la materia en el plan de estudios y equipamiento disponible.

El eje fundamental de todo entorno que propongamos es la participación activa de todos a diferentes niveles de implicación, en un ambiente abierto y sin restricciones a la participación. Se garantiza así una comunidad discursiva y de aprendizaje. Dada las características de la 
población que se analiza, se plantea una relación horizontal en que el liderazgo es compartido entre los directivos de la red. Aun así, es necesario contemplar el concepto de liderazgo, al menos con el fin de efectuar la tarea de evaluación y seguimiento de las tareas realizadas. Por supuesto, se requiere de participación voluntaria y gratuita. Si bien, ello hace a la descripción del medio interno, la red puede solicitar apoyo a una amplia variedad de profesionales.

Las alternativas posibles de plantearse, permiten que se pueda participar en tareas en el mismo momento independientemente del lugar que se encuentren (sincrónico), o bien la realización de trabajo en el tiempo particular de cada uno (asincrónico). Dado el carácter nacional de la población, distribuido en casi todas las provincias, la red será accesible sin limitaciones geográficas y lógicamente desde las propias computadoras personales. Por otra parte, los miembros de la red son de alta estabilidad, contrariamente a lo que ocurriría en una red de alumnos, con todo lo que ello implica en cuanto a organización, planificación de tareas, etc.

Un motivo importante para el trabajo colaborativo en cuestión de contenidos, o curriculas de las materias es la alta tasa de movilidad del alumnado en cuanto a programas de intercambio nacional e internacional. En la medida en que se cuente con programas con algún grado de consenso entre las universidades, se le facilitaría a los interesados participar en estos programas al poder obtener la aprobación de las materias cursándolas en otras entidades elegidas por ellos. Lo mismo vale para cuestiones de pedidos de equivalencias por motivos de cambios de universidad, etc.

Cualquiera de las asociaciones docentes que mencionamos, podría llevar adelante esta implementación de trabajo interactivo, dadas las características similares a la población analizada. 
Las plataformas basadas en computación en la nube en que interactúan actualmente estas entidades, cuentan con las herramientas sincrónicas y asincrónicas antes citadas, suficientes para tratar la problemática descrita y llevar adelante un trabajo conjunto.

\section{V.2. Caso 2 - Investigación Colaborativa}

La investigación colaborativa tiene una característica esencial y es la del trabajo en grupo, es de hecho una de las formas más adecuadas de construir una comunidad científica y obtener datos e información desde donde se pueda lograr un conocimiento para luego ser aprovechado por el mismo grupo y la sociedad. La colaboración no es un fin en sí mismo, pero sí un medio para alcanzar ciertos objetivos, superándose así el aislamiento investigador del profesor y conformándose un grupo como marco investigador.

La investigación colaborativa se refiere a los casos en que varias entidades comparten el interés por la ejecución de un proyecto, el esfuerzo por desarrollarlo, los riesgos y la propiedad de los resultados conforme con su diversa contribución para obtenerlos. Una investigación es colaborativa cuando un grupo de personas se ponen de acuerdo en buscar algo en común, y esta búsqueda la desarrollan de una manera articulada, cooperativa, coordinada y orientando sus fuerzas y conocimientos hacia el objetivo establecido.

Algunas características necesarias son: deseo de trabajar en equipo, participación colaborativa, formación de lazos sociales, esfuerzos grupales para la elección del tema y la resolución del problema.

La colaboración ofrece importantes ventajas, que la convierten en un valioso recurso. Son varias las razones para que eso se verifique:

- Al unir diversas personas con experiencias, competencias y perspectivas diversas, se reúnen más recursos para concretar, con éxito, un determinado trabajo, dándose de este modo un aumento de seguridad para promover cambios e iniciar innovaciones. 
- Al unir diversas personas que interactúan, dialogan y reflexionan en conjunto, se crean sinergias que posibilitan una gran capacidad de reflexión y un aumento de las posibilidades de aprendizaje mutuo, permitiendo, así, ir mucho más lejos y crear mejores condiciones para enfrentar, con éxito, las incertidumbres y los obstáculos que surjan.

La utilización del término colaboración es adecuada en los casos en que los diversos participantes trabajan de manera conjunta, no en una relación jerárquica, sino sobre una base de igualdad, de modo que haya una ayuda mutua para alcanzar los objetivos que beneficien a todos. Un trabajo colaborativo no depende sólo de la existencia de un objetivo general común. Las formas de trabajo y de relación entre los miembros del equipo tienen que ser propiciadoras del trabajo conjunto. Subyacente a la idea de la colaboración está una cierta mutualidad en la relación: todos tienen algo para dar y algo para recibir del trabajo conjunto. Significa que todos los participantes tienen un papel reconocido en el proyecto y se benefician con su contribución (Boavida y Da Ponte, 2011). Un participante en un proyecto de investigación colaborativa tiene que asumir un mínimo de protagonismo, no reduciéndose, por ejemplo, su papel al de un simple proveedor de datos a otros participantes.

Uno de los retos que se manifiestan en la mayor parte de las instituciones educativas con respecto a la actuación de los profesores, es la de incorporar la investigación como una actividad sistemática y regular en su trabajo habitual en las aulas. La investigación como un procedimiento y un medio de desarrollo y aplicación del conocimiento, se ha configurado como una forma de trabajo. El compromiso de la investigación con relación al avance del conocimiento y su aplicación, ha identificado dos estilos de indagación como tendencias: la investigación básica cuya pretensión es asegurar el conocimiento pero sin una relación directa o compromiso alguno con su aplicación en problemas prácticos o de modificación de la realidad y como complemento de esta, la investigación aplicada se caracteriza por su asociación directa en la solución de los problemas reales planteados en relación con el hombre y sus diversas acciones. 
Una prueba de la creciente integración en el ámbito académico basado en computación en la nube, es que los programas informáticos que se utilizan actualmente en administración de bases de datos bibliográficas, cuentan en sus funciones con herramientas para trabajo colaborativo. Es así que estos programas permiten invitar a colegas a acceder a la bibliografía propia o compartir las investigaciones en curso.

\section{V.3. Caso 3 - Herramientas de Medición y Optimización de la Gestión Educativa}

La creación de sistemas que permitan medir la eficiencia de los equipos de trabajo puede ser un aliciente para su éxito. Actualmente, las universidades aplican la metodología de encuestas para evaluar el desempeño académico de sus docentes, pero se hace en forma no integrada. Cada cátedra es calificada por el alumnado pero los resultados solo analizado individualmente. Cada profesorado se preocupa por alcanzar los objetivos pedagógicos propios, pero la visión conjunta es habitualmente dejada de lado. Las nuevas tecnologías de la información y la comunicación, principalmente audiovisual, informática, telemática y multimedia, permiten repensar los sistemas de enseñanza presenciales y no presenciales y potenciarlos. En esta línea de análisis, puede pensarse en la comparación de encuestas de evaluaciones docentes entre diferentes Facultades o incluso universidades. En algunos países, es habitual su publicación y amplia difusión de los resultados, como medio para mostrar las evaluaciones que hacen sus alumnos de los servicios que reciben.

Las encuestas de evaluación de docentes incluyen preguntas habituales y, aunque su interpretación literal puede ser diversa en distintos lugares, normalmente evalúan: a) conocimiento del tema; b) didáctica en la clase; c) asistencia y puntualidad; d) material complementario utilizado (condicionado por el medio de enseñanza); e) forma, medio y tiempo adecuado en las evaluaciones a los alumnos. Si todo esto es cierto, se podrían integrar dichos ítems, al menos por aproximación, con resultados de objetivos colectivos acordes con los objetivos institucionales. 
Como ejemplo de dichos objetivos, la investigación educativa podría intentar medir la reducción de la deserción y el número de aplazados derivado de la aplicación de distintas combinaciones de recursos pedagógicos en las disciplinas de administración de empresas de distintas universidades del país, buscando reducir el número de desaprobados, manteniendo constante la calidad educativa. Ahora bien, la fijación de objetivos es un camino en la dirección de establecer mecanismos para medición de la eficiencia de la labor en equipo.

La investigación consiste en la búsqueda de una combinación de herramientas y materiales didácticos para entornos virtuales y presenciales de enseñanza - aprendizaje, partiendo de una concepción interactiva. El producto final sería la conformación, diseño y uso de esa combinación de recursos, normas y enfoque educativo adecuado, con la posibilidad de diferenciar asignaturas en función del nivel introductorio/avanzado, grado/posgrado, etc.

Analizar el rendimiento estudiantil es necesariamente ejemplo de interacción docente, dado que implica trabajo interdisciplinario, lo que es sinónimo de trabajo en equipo interactivo. Más aún, todos los programas actuales efectúan mediciones comparativas entre distintas universidades, países, clases sociales, etc., lo que conlleva a un necesario trabajo en la nube.

\section{Discusión}

En todos los ámbitos que se planteó la temática mientras se elaboraba el documento, reuniones de discusión y jornadas docentes, la misma resultó ciento por ciento atractiva para quienes participaron en la presentación y consideraron aplicable la propuesta hacia distintos ámbitos, ya sea, institutos o departamentos docentes, asociaciones docentes, cátedras, etc. Las dificultades del trabajo en equipo que se mencionaron no parecen representar una limitación para el ímpetu actual en el trabajo colaborativo.

Si bien, en la investigación no se emplearon técnicas cuantitativas para evaluación de posibles resultados y del estado actual, ello obedece a que el objetivo no es la descripción estadística sino promover su implementación. La medición de resultados de trabajo en equipo docente no 
es cuantificable de un modo convencional, como lo sería la medición de costos o beneficios. Pero queda planteado que el diseño de dichos indicadores, requiere en primer término la implementación de la política propuesta y la definición de los objetivos por evaluar. En otras palabras, el foco fue puesto en analizar los aspectos blandos del trabajo en equipo en la nube, acorde con los objetivos propuestos.

Si bien la temática de trabajo en equipo ha sido ampliamente tratada en investigación científica, la integración con los temas que aporta el presente trabajo resulta una combinación inédita. Primero, porque la nube potencia práctica y emocionalmente al participante. Segundo, porque materializa la aplicación a casos concretos de entidades que desempeñan un rol central en interacción docente actualmente. Tercero, porque integra aspectos informáticos y habilidades humanas.

Los casos planteados describen distintas situaciones y si bien en las conclusiones oportunamente se plantearon las particularidades de cada caso, todos ellos unívocamente conducen hacia las ventajas de la interacción en la nube descritas en el trabajo, prácticamente sin contraindicaciones, en términos de administración: ganar-ganar.

Instituciones, universidades e investigadores que tengan previsto profundizar estudios sobre la materia, deberían paralelamente poner en práctica un trabajo colaborativo docente que retroalimente la investigación. En tal sentido, hemos planteado ejemplos replicables a distintas situaciones académicas, que podrán servir de base a dicha implementación junto a este documento de apoyo; es decir, su transferibilidad.

\section{Conclusiones}

1. Las redes de profesores contribuyen a mejorar la calidad de la enseñanza, ya que permiten a los docentes expandir su conocimiento en una gran cantidad de dimensiones: contenido pedagógico, currículo y contexto educativo, características de los estudiantes, objetivos educativos y propósitos. 
2. El potencial de las redes de profesorado está actualmente limitado por la escasa interoperatividad entre ellas. Para aprovechar los potenciales beneficios de las redes, deberían ponerse en práctica políticas que promuevan soluciones de computación en la nube.

3. Los asuntos relativos a la privacidad constituyen uno de los principales desafíos de las plataformas de redes. Sin embargo, las razones para la protección de la privacidad no deberían ser argumentadas para defender sistemas cerrados. Asimismo, sería adecuado desarrollar soluciones que aseguren que los miembros de las redes puedan controlar sus datos personales.

4. La consolidación y difusión de las redes de profesores es un objetivo primordial. Para alcanzarlo debe promocionarse la motivación extrínseca de los docentes a través de un entorno organizativo de apoyo; por ejemplo, estableciendo planes de reconocimiento del desarrollo profesional de los profesores.

5. Según Bacigalupo y Cachia (2011) estas redes evolucionarán en paralelo con las TICs, se harán tan cruciales que las comunidades educativas participarán en el diseño de futuras aplicaciones que servirán a sus necesidades y determinarán la manera de llevar a cabo sus actividades. El futuro apunta en esta dirección.

\section{Referencias}

Area, M. (2009). Introducción a la tecnología educativa. Universidad de La Laguna. Recuperado de https://manarea.webs.ull.es/wp-content/uploads/2010/06/ebookte.pdf

Arroyo, N. (2007). ¿Web 2.0? ¿Web Social? ¿Qué es eso? Revista Educación y Biblioteca, $161,69-74$

Bacigalupo, M., y Cachia, R. (2011). Teacher colaboration networks in 2025. Notas del taller realizado el 6 y 7 de Junio en el Institute for Prospective Technological Studies of the European Commission Joint Research Centre. Recuperado de http://ftp.jrc.es/EURdoc/JRC67530_TN.pdf 
Boavida, A. y Da Ponte, J. (Enero-Abril, 2011). Investigación colaborativa: Potencialidades y Problemas. Revista Educación y Pedagogía, 23(59), 125-135.

Cisco. (2010). Informe Técnico. Computación en la nube para la educación superior: guía de evaluación y adopción. Recuperado de

http://www.cisco.com/web/LA/soluciones/strategy/education/connection/pdfs/Cisco_Ca mpus_Technology_Whitpaper.pdf

Feldman, G. y Juarez, E. (2014). Interacción docente en la nube, aplicada a finanzas. XXXIV Jornadas de la Sociedad Argentina de Docentes en Administración Financiera. Córdoba. ISBN 2362-4728. 63-78

Joyanes, L. (2012). Computación en la nube. Notas para una estrategia española en cloud computing. Revista del Instituto Español de Estudios Estratégicos, 0, 89-112.

Larios, Y. (2014). Guía metodológica para la protección de datos en la utilización de la computación en la nube. (Tesis especialización en auditoría de sistemas de información). Corporación Universidad de la Costa. Colombia

Laudon, K., y Laudon, J. (2012). Sistemas de Información Gerencial. 12a Ed. México: Pearson

Lobato, C. y Madinabeitia, A. (2011). Perfiles motivacionales del profesorado ante la formación en metodologías activas en la universidad. Revista Formación Universitaria, 4(1), 37-48

Medina, G. (2014). Sistema de información gerencial. Comercio electrónico global y colaboración. Recuperado de: http://grupo6-jgr.blogspot.com.ar/2014/07/comercioelectronico-global-y.html

Montoya, M., Gil, C. et al. (2008). El trabajo en equipo y la coordinación del profesorado. III Jornadas sobre el espacio europeo en educación superior: "Avanzando hacia Bolonia". Murcia, 8 y 9 de mayo.

Rodas, F. y Toscano, D. (2015). Propuesta de un modelo de gestión de servicios de tecnologías de información y comunicación en la nube (cloud computing) para universidades. (Tesis de maestría de Magister en gestión de las comunicaciones y tecnologías de la información). Escuela Politécnica Nacional. Ecuador.

Pere, G. (2013). La Web 2.0 y sus aplicaciones didácticas. Recuperado de http://peremarques.pangea.org/web20.htm 